\title{
An ultrasound-guided fascia iliaca catheter technique does not impair ambulatory ability within a clinical pathway for total hip arthroplasty
}

\author{
Seshadri C. Mudumbai ${ }^{1,3}$, T. Edward Kim ${ }^{1,3}$, Steven K. Howard ${ }^{1,3}$, \\ Nicholas J. Giori ${ }^{2,3}$, Steven Woolson ${ }^{2,3}$, Toni Ganaway ${ }^{1}$, Alex Kou ${ }^{1}$, \\ Robert King ${ }^{3}$, and Edward R. Mariano ${ }^{1,3}$ \\ Departments of ${ }^{1}$ Anesthesiology, Perioperative and Pain Medicine, ${ }^{2}$ Orthopaedic Surgery, Stanford University \\ School of Medicine, Stanford, ${ }^{3}$ Veterans Affairs Palo Alto Health Care System, Palo Alto, CA, USA
}

Background: Both neuraxial and peripheral regional analgesic techniques offer postoperative analgesia for total hip arthroplasty (THA) patients. While no single technique is preferred, quadriceps muscle weakness from peripheral nerve blocks may impede rehabilitation. We designed this study to compare postoperative ambulation outcome in THA patients who were treated with a new ultrasound-guided fascia iliaca catheter (FIC) technique or intrathecal morphine (ITM). Methods: We reviewed the electronic health records of a sequential series of primary unilateral THA patients who were part of a standardized clinical pathway; apart from differences in regional analgesic technique, all other aspects of the pathway were the same. Our primary outcome was total ambulation distance (meters) combined for postoperative days 1 and 2. Secondary outcomes included daily opioid consumption (morphine milligram equivalents) and analgesic-related side effects. We examined the association between the primary outcome and analgesic technique by performing crude and adjusted ordinary least-squares linear regression. A P value $<0.05$ was considered statistically-significant.

Results: The study analyzed the records of 179 patients (fascia iliaca, $n=106$; intrathecal, $n=73$ ). The primary outcome (total ambulation distance) did not differ between the groups $(\mathrm{P}=0.08)$. Body mass index (BMI) was the only factor $(\beta$ $=-1.7[95 \% \mathrm{CI}-0.5$ to -2.9$], \mathrm{P}<0.01$ ) associated with ambulation distance. Opioid consumption did not differ, while increased pruritus was seen in the intrathecal group $(\mathrm{P}<0.01)$.

Conclusions: BMI affects postoperative ambulation outcome after hip arthroplasty, whereas the type of regional analgesic technique used does not. An ultrasound-guided FIC technique offers similar analgesia with fewer side effects when compared with ITM.

Key Words: Hip arthroplasty, Hip replacement, Nerve block, Regional anesthesia, Spinal anesthesia, Ultrasonography.

Corresponding author: Edward R. Mariano, M.D., M.A.S.

Department of Anesthesiology, Perioperative Care Service, Veterans Affairs Palo Alto Health Care System, 3801 Miranda Avenue (112A), Palo Alto, CA 94304, USA

Tel: 1-650-849-0254, Fax: 1-650-852-3423

E-mail: emariano@stanford.edu

ORCID: http://orcid.org/0000-0003-2735-248X

Received: November 19, 2015. Revised: December 29, 2015. Accepted: February 14, 2016.

Korean J Anesthesiol 2016 August 69(4): 368-374

http://dx.doi.org/10.4097/kjae.2016.69.4.368

(c) This is an open-access article distributed under the terms of the Creative Commons Attribution Non-Commercial License (http://creativecommons.org/ licenses/by-nc/4.0/), which permits unrestricted non-commercial use, distribution, and reproduction in any medium, provided the original work is properly cited. 


\section{Introduction}

Every year, approximately 300000 patients undergo total hip arthroplasty (THA) in the United States [1]. Clinical care pathways have been increasingly used to apply evidence-based approaches for the perioperative management of these patients, to facilitate rehabilitation and improve outcomes [2]. Standardized protocols for the pre-, intra-, and postoperative periods have demonstrated improvements in time to functional recovery, particularly in early postoperative ambulation. Early ambulation after THA helps to decrease deep venous thrombosis of the legs, enhance muscle strength and gait control, and shortens the length of hospital stay [3-5].

Central to the multimodal analgesic regimen of common THA clinical pathways is the use of regional analgesia, and both continuous peripheral nerve block (CPNB) and neuraxial techniques have been described [6,7]. CPNB techniques involve the percutaneous placement of a catheter near a target nerve or plexus (i.e., a perineural catheter) for continuous local anesthetic infusions affecting only the side of surgery. The fascia iliaca compartment containing the femoral and lateral femoral cutaneous nerves is used as a site for perineural catheters in THA patients [8]. Although not selective for the operative side, neuraxial techniques have been associated with less perioperative hypercoagulability and a reduced surgical neuroendocrine stress response, and intrathecal opioids such as morphine provide effective pain control during the immediate postoperative period [9].

The relative effectiveness of these two regional analgesic techniques for promoting early ambulation after THA is not clear [10], although CPNB techniques involving the femoral nerve have been associated with quadriceps muscle weakness [11]. We hypothesized that THA patients who underwent spinal anesthesia with a single dose of intrathecal morphine (ITM) would achieve a greater combined ambulation distance on postoperative days (POD) 1 and 2 when compared with patients who had an ultrasound-guided fascia iliaca catheter (FIC) with perineural local anesthetic infusion.

\section{Materials and Methods}

This study was performed at a tertiary care university-affiliated Veterans Affairs (VA) medical center. We conducted this retrospective cohort study after receiving both university Institutional Review Board approval, with a waiver of informed consent, and local study approval from the VA research committee.

Using a custom-made perioperative database [12], we identified a sequential series of primary unilateral THA patients treated from 2012 to 2014 who received multimodal analgesia within a clinical pathway that was standardized in all aspects, except for the choice of regional analgesic technique (Table 1). Patients were included if they: 1) underwent unilateral THA during the study period; 2) received either ultrasound-guided FIC or ITM; and 3) were admitted to the primary surgical ward postoperatively. Patients were excluded it they underwent surgical procedures in addition to unilateral THA, were treated with a different regional analgesic technique, or lacked documentation on the ambulation distance for either POD 1 or 2 . We collected baseline biometric, perioperative medication, and administrative data from our VA electronic health records.

The multimodal analgesic regimen was similar to one published for total knee arthroplasty, except for the site of regional analgesia [13]; for THA patients at our institution during the study period, since this was not a prospective study, the choice of regional analgesic technique was left to the discretion of the anesthesiologist in collaboration with the surgeon and patient. Apart from the choice of regional analgesic technique, the clinical analgesic pathway for FIC and ITM patients had no other differences during the study period.

\section{Ultrasound-guided FIC technique}

In the FIC group, all patients underwent preoperative ultrasound-guided insertion of a perineural catheter. These procedures were performed by either an attending regional anesthesiologist or a clinical regional anesthesiology and acute pain medicine fellow, supervised one-on-one by an attending regional anesthesiologist. Patients, who were monitored according to the American Society of Anesthesiologists (ASA) standards, received

Table 1. Analgesic Clinical Pathway for Total Hip Arthroplasty

\begin{tabular}{ll}
\hline Preoperative & Insertion of a fascia iliaca perineural catheter or spinal with bupivacaine $0.75 \%$ and preservative-free morphine $0.2 \mathrm{mg}$ \\
Intraoperative & Periarticular local anesthetic infiltration by the surgeon of $100-150 \mathrm{ml}$ of $0.2 \%$ ropivacaine containing epinephrine with ketorolac \\
& $30 \mathrm{mg}$ at the end of surgery \\
Postoperative & - Perineural infusion of $0.2 \%$ ropivacaine at $6 \mathrm{ml} / \mathrm{h}$ with a patient-controlled bolus of $5 \mathrm{ml}$ ( 30 minute lockout) when applicable \\
& - Scheduled oral medications: oxycodone, acetaminophen, and diclofenac \\
& - Breakthrough analgesics: oxycodone (oral, first line) and hydromorphone (intravenous, second line) for pain not relieved by \\
scheduled medications & - No routine intravenous opioid patient-controlled analgesia \\
\hline
\end{tabular}


intravenous sedation during the procedure, titrated to comfort while maintaining verbal responsiveness. Using sterile techniques, the femoral nerve was visualized in the short-axis with a high-frequency 6-13 MHz ultrasound transducer (HFL38, MTurbo, Fujifilm SonoSite, Bothell, WA, USA), and the placement needle was guided out-of-plane toward the fascia iliaca lateral to the femoral nerve after infiltrating the needle-insertion site with local anesthetic (Fig. 1A). After penetrating the fascia iliaca, the fascia was separated from the iliacus muscle using a hydrodissection technique with mepivacaine $1.5 \%$. Next, the transducer was rotated $90^{\circ}$ to visualize the needle in the long-axis. The placement needle was advanced incrementally in a cephalad and medial direction with intermittent local anesthetic injection using a long-axis in-plane technique (Fig. 1B). The total volume of local anesthetic administered was 30-40 ml. A non-stimulating flexible epidural-type catheter (Arrow FlexTip Plus, Teleflex Medical, Research Triangle Park, NC, USA) was advanced 5-10 $\mathrm{cm}$ beyond the placement needle tip. In the post-anesthesia care unit, the catheter was attached to a portable infusion device (ON-Q C-bloc with ONDEMAND ${ }^{\mathrm{TM}}$; Halyard Health, Lake Forest, CA, USA) set to deliver an infusion of $0.2 \%$ ropivacaine (basal rate $6 \mathrm{ml} / \mathrm{h}$; patient-controlled bolus of $5 \mathrm{ml}$; and 30-minute lockout interval).

\section{ITM technique}

The ITM was administered preoperatively with the spinal anesthetic upon initial arrival in the operating room. Patients were seated according to standard ASA monitoring. After palpating a suitable lumbar spinal interspace using surface landmarks and a sterile technique, the needle-insertion site was infiltrated with local anesthetic followed by placement of the introducer needle.
A 25-gauge $90 \mathrm{~mm}$ Whitacre needle (B. Braun, Bethlehem, PA, USA) was advanced through the introducer needle until it entered the dural sac, as confirmed by the free flow of cerebrospinal fluid. A single dose of morphine (typically $0.2 \mathrm{mg}$ ) was injected intrathecally, along with a volume of $0.75 \%$ bupivacaine according to the discretion of the anesthesiologist.

Intraoperatively, although most patients received general anesthesia, no standardized anesthetic technique was promoted. All patients underwent a primary THA through a posterolateral approach. Upon reduction of the new prosthetic hip and before tissue closure, the surgeons infiltrated the region around the joint and surrounding tissues evenly with local anesthetic $(0.2 \%$ ropivacaine containing epinephrine [100-150 ml] and ketorolac $30 \mathrm{mg}$ ). Postoperatively, all patients received the same multimodal analgesic regimen as per protocol (Table 1).

Routine postoperative care on the surgical ward included a standardized physical therapy regimen. Starting on the morning of POD 1, patients began weight bearing as tolerated and underwent twice-daily physical therapy sessions consisting of transfers and ambulation with progression to stair climbing. Patients ambulated with the assistance of a front-wheel walker. All FIC infusions were routinely stopped at 5 a.m. each day and resumed after the conclusion of the afternoon physical therapy session.

Our primary outcome was total ambulation distance (meters) combined for both POD 1 and 2. Secondary outcomes included: a) the total daily opioid consumption (morphine milligram equivalents) on POD 1 and 2 recorded using barcode administration records; b) pain with movement assessed by the physical therapist twice daily on POD 1 and 2 using a numeric rating scale (NRS; 0 = no pain; 10 = worst possible pain); c) any episode of postoperative nausea and vomiting (PONV) on POD 1; and d) any complaint of pruritus on POD 1.
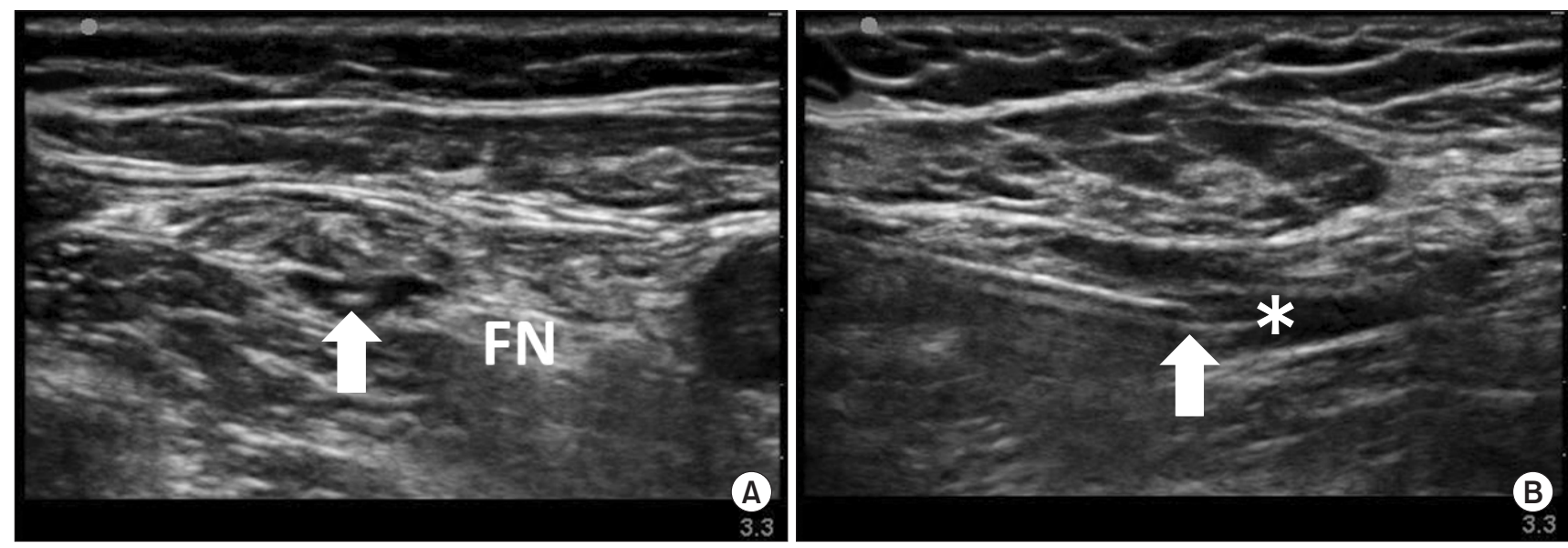

Fig. 1. (A) Short-axis sonogram demonstrating the initial out-of-plane needle insertion. (B) Long-axis in-plane view after the transducer is rotated 90 degrees; the catheter is inserted into the fascia iliaca compartment after distention with injectate and advancement of the needle. Arrow: needle tip, *atheter tip, FN: femoral nerve. 


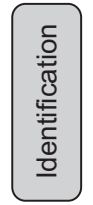

183 Patients with unilateral primary total hip arthroplasty (2012-2014)

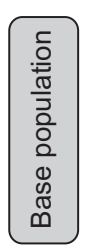

- 4 Patients who lacked documentation on ambulation distance on postoperative day 1 or 2 (fascia iliaca

catheter $=3$, intrathecal morphine $=1$ )

Fascia iliaca catheter $(n=106)$
Intrathecal morphine $(n=73)$

\section{Statistical analysis}

First, descriptive statistics were examined and the extent of missing data was evaluated. Normality of distribution was determined using the Kolmogorov-Smirnov test. Bivariate comparisons involved the use of standard parametric (i.e., Student's t-test for normal distributions) or nonparametric (Wilcoxon rank-sum test for non-normal distributions) tests for continuous variables. For categorical variables, the chi-square test or Fisher's exact test was used ( $<5$ in any field). All opioids were converted into morphine dosage equivalents, and the total doses received on POD 1 and 2 were calculated. Then, we examined the association between the primary outcome and analgesic technique by performing crude and adjusted ordinary least-squares linear regression. All potential confounders were determined a priori and were forced into the linear model. Potential confounders included age, gender, height, weight, body mass index (BMI), ASA physical status classification, and operating surgeon. All P values were two-sided; a P value of less than 0.05 was considered statistically significant. STATA software (ver 12.1; STATA Corp., College Station, TX, USA) was used for all analyses.

\section{Results}

Of the 183 potentially eligible patients in the sequential series, four were excluded because of a lack of documentation of
Fig. 2. Study flow diagram.

Table 2. Patient Characteristics

\begin{tabular}{lccc}
\hline & $\begin{array}{c}\text { Fascia iliaca } \\
\text { catheter } \\
(\mathrm{n}=106)\end{array}$ & $\begin{array}{c}\text { Intrathecal } \\
\text { morphine } \\
(\mathrm{n}=73)\end{array}$ & P value \\
\hline Age $(\mathrm{yr})$ & $66.0(9.3)$ & $63.4(9.0)$ & 0.06 \\
Sex $(\mathrm{M} / \mathrm{F}, \mathrm{n})$ & $100 / 6$ & $69 / 4$ & 0.96 \\
Height $(\mathrm{m})$ & $1.8(0.1)$ & $1.7(.1)$ & 0.48 \\
Weight $(\mathrm{kg})$ & $96.1(22.1)$ & $95.5(22.5)$ & 0.88 \\
Body mass index $\left(\mathrm{kg} / \mathrm{m}^{2}\right)$ & $30.9(7.3)$ & $31.1(6.3)$ & 0.89 \\
ASA classification $(\mathrm{n})$ & & & \\
$\quad 1$ & 0 & 0 & $>0.99$ \\
2 & 19 & 21 & 0.10 \\
3 & 85 & 52 & 0.21 \\
4 & 2 & 0 & 0.51 \\
Length of stay (days) & $4.7(3.4)$ & $4.2(2.7)$ & 0.31 \\
\hline
\end{tabular}

Data are presented as means (SD) or as number of subjects (n), as appropriate. ASA: American Society of Anesthesiologists.

postoperative ambulation for POD 1 or 2 (FIC, $\mathrm{n}=3$; ITM, $\mathrm{n}=1$ ). The final study sample included 179 patients: 106 in the FIC group and 73 in the ITM group (Fig. 2). Table 2 summarizes the key patient characteristics of each group. The two groups were comparable in age, sex, height, weight, BMI, ASA physical status, and length of stay.

The primary outcome, total ambulation distance in meters (median [Q1-Q3]), did not differ between the two groups (FIC 63 [30-120] vs. ITM 83 [48-1114]; P = 0.08). The adjusted linear 
Table 3. Secondary Outcomes

\begin{tabular}{lccc}
\hline & Fascia iliaca catheter $(\mathrm{n}=106)$ & Intrathecal morphine $(\mathrm{n}=73)$ & P value \\
\hline Total opioid use POD 1 (mg MS) & $30.0(22.5-45.0)$ & $45.0(30.0-52.5)$ & 0.07 \\
Total opioid use POD 2 (mg MS) & $57.8(30.0-60.0)$ & $60.0(43.5-79.5)$ & 0.06 \\
Postoperative nausea and vomiting on POD 1 (n) & 12 & 15 & 0.14 \\
\hline
\end{tabular}

Data are presented as medians (Q1-Q3) or as number of subjects (n), as appropriate. mg MS: morphine milligram equivalents, POD: postoperative day.

regression maintained our findings. BMI was negatively associated with the total ambulation distance in meters for POD 1 and 2 in the linear model $(\beta=-1.7$ [95\% CI -0.5 to -2.9 ], $\mathrm{P}<0.01$, adjusted $\mathrm{R}^{2}=0.64$ ).

Many physical therapy notes lacked pain score documentation. On the morning of POD 1, pain with movement was 4.5 [2.0-6.5] for FIC $(n=93)$ vs. 4.3 [2.3-6.9] for ITM $(n=70$; $=$ $0.98)$. In the afternoon, pain with movement for FIC $(\mathrm{n}=41)$ was $2.5[0.0-5.0]$ vs. 4.3 [2.9-7.0] for ITM $(\mathrm{n}=28 ; \mathrm{P}=0.01)$. On POD 2, the morning movement pain scores were 2.5 [0.3-5.0] for FIC $(n=79)$ vs. 4.5 [2.1-6.0] for ITM $(n=50 ; P=0.02)$; the afternoon scores were 3.8 [1.1-5.0] for FIC $(n=30)$ vs. $3.3[0.0-$ 5.9] for ITM ( $\mathrm{n}=16 ; \mathrm{P}=0.94)$. The total daily opioid consumption and PONV did not differ between the two groups (Table 3). However, pruritus on POD 1 was reported by more patients in the ITM group compared with the FIC group ( $n=9 / 73$ vs. $n=$ 2/106, respectively; $\mathrm{P}=0.008$ ).

\section{Discussion}

This single-center retrospective cohort study found no overall advantage for either ultrasound-guided FIC or ITM in terms of early postoperative ambulation or opioid consumption after THA. In fact, BMI was more strongly associated with immediate postoperative ambulation after this surgery than was the analgesic technique. As BMI increases, ambulation decreases in a linear fashion. Given ongoing interest in promoting functional outcomes after joint replacement, this study clarifies the role of two different analgesic techniques in the context of established clinical pathways [10]. Our study is one of the first to provide data comparing the effectiveness of these two analgesic techniques in the context of THA patients and to evaluate the potential benefits and side effects of each in a "real-world" clinical setting [14].

Our results are clinically relevant given the growing prevalence of obesity in the United States, the increasing number of lower extremity joint arthroplasty surgeries, and increasing emphasis on early mobilization for these patients [15-17]. For obese or morbidly obese patients (i.e., BMI $>40 \mathrm{~kg} / \mathrm{m}^{2}$ ), early ambulation after joint replacement may be problematic regardless of the effectiveness of pain management [18,19]. Early ambulation after THA has been promoted for more rapid restoration of normal hip motion and strength, along with a quicker return to everyday activities. In turn, increased hip strength may also correlate with reduced falls and decreased morbidity [20,21]. However, recent data suggest that obese and morbidly obese patients have poorer skeletal muscle quality and experience fear of movement and avoidance of physical activities, resulting in decreased functional outcomes $[18,22,23]$. In clinical practice, therefore, we may need to: 1) identify patients with increased BMI early in the preoperative evaluation process; 2) encourage prehabilitation (i.e., preoperative strength training to enhance functional capacity), weight loss, and healthy eating; and 3) allocate additional resources and personnel as needed to facilitate aggressive early postoperative rehabilitation $[17,23,24]$.

The efficacy of a single-injection fascia iliaca block for postTHA pain has been questioned [25], but a randomized study showed that a stimulating femoral catheter technique that is functionally similar to our ultrasound-guided FIC technique provides equivalent analgesia to posterior lumbar plexus perineural infusion for THA patients [26]. The use of CPNB has been shown to decrease the time to discharge eligibility for THA patients when compared with a placebo control [27]. Our results suggest that there is an advantage, in terms of movement-related pain control, to using FIC in the afternoon of POD 1 and in the morning of POD 2, consistent with the resolution of ITM analgesia. However, we advise caution in interpreting these results given the amount of missing data and lack of a difference in opioid consumption; a prospective study is clearly warranted.

This study found no advantage in terms of ambulation achievement or opioid consumption between FIC and ITM, although more ITM patients complained of pruritus on POD 1. A lack of superiority should not be confused with equivalence, but it is interesting that patients with FIC in our study do not appear to suffer from greater physical impairment when compared with ITM patients. We believe that proper management of the FIC is the key. Since every FIC infusion is routinely stopped early each morning, we speculate that our FIC patients are able to recover sufficient quadriceps muscle strength to perform physical therapy activities such as ambulation, unlike subjects from previous studies involving uninhibited continuous perineural local anesthetic infusions for THA patients [27]. Given the lack of superi- 
ority in terms of achieving ambulation, either technique may be acceptable as the regional analgesic component of a multimodal analgesic protocol. Other factors may also influence the choice of regional analgesic technique (e.g., anticoagulant or antiplatelet drugs [28], previous spine surgery, the training of the anesthesiologist in regional anesthesia, or systems issues), and the choice may vary on a patient-to-patient basis. For intraoperative anesthesia, spinal anesthesia with or without general anesthesia offers advantages in terms of decreasing transfusion requirements and all-cause postoperative morbidity when compared with general anesthesia alone for THA patients [29]. While FIC approaches have demonstrated benefits for patients undergoing hip fracture repair, more research is needed on both pain management and functional outcomes for THA patients using the FIC technique [26,30].

This study had several limitations. First, conclusions regarding causality should be drawn cautiously because the study is retrospective [14]. However, our study focuses on the immediate postoperative period when the analgesic benefits and side effects of CPNB catheters are most relevant. Second, given that this is a retrospective study and therefore not randomized, selection bias based regarding patient characteristics or operating room logistics may have affected how patients were treated in the clinical setting. We have attempted to minimize this bias by including sequential surgical patients over a 2-year timeframe. Our patient sample also showed no differences in key baseline characteristics by group. We examined patients who were managed as part of a clinical pathway with all other aspects of the clinical pathway re- maining constant during this time period (e.g., physical therapy regimen, nursing care, and analgesic medications). Therefore, the strengths of this study include the examination of actual patients in a real clinical setting, instead of healthy volunteers or "ideal" clinical research participants. Third, our results should be interpreted within the context of an established clinical pathway and therefore may not be applicable to hospitals not following a similar clinical pathway. Fourth, our study was conducted at a single, university-affiliated VA medical center with associated idiosyncrasies (e.g., male-dominated patient population, graduate medical education) and so may not be generalizable to every institution. However, the length of observation under conditions of routine clinical practice and management by multiple surgeons, a single surgery type, and a cohort consisting of a sequential series of patients support the external validity of our study results. Finally, our study was not designed to examine differences in the secondary outcomes. Therefore, the results of these analyses should be interpreted as suggestive and not conclusive.

In summary, this study found no overall advantage in favor of ultrasound-guided FIC or ITM in terms of ambulation achievement or opioid consumption on POD 1 and 2 after THA. A regional analgesic technique is a key component of the multimodal analgesic protocol for lower extremity total joint replacement patients, but this study does not support a preferred technique for THA patients. Our data suggest that other factors, such as BMI, affect postoperative ambulation more than analgesic technique for THA patients [18].

\section{References}

1. Merrill C, Elixhauser A. Hospital Stays Involving Musculoskeletal Procedures, 1997-2005 [Internet]. Statistical Brief \#34. Healthcare Cost and Utilization Project (HCUP) Statistical Briefs. Rockville (MD), 2006. Available from http://www.ncbi.nlm.nih.gov/books/ NBK61973/?report=printable

2. Barbieri A, Vanhaecht K, Van Herck P, Sermeus W, Faggiano F, Marchisio S, et al. Effects of clinical pathways in the joint replacement: a meta-analysis. BMC Med 2009; 7: 32.

3. Bernucci F, Carli F. Functional outcome after major orthopedic surgery: the role of regional anesthesia redefined. Curr Opin Anaesthesiol 2012; 25: 621-8.

4. Hebl JR, Dilger JA, Byer DE, Kopp SL, Stevens SR, Pagnano MW, et al. A pre-emptive multimodal pathway featuring peripheral nerve block improves perioperative outcomes after major orthopedic surgery. Reg Anesth Pain Med 2008; 33: 510-7.

5. Napier RJ, Spence D, Diamond O, O'Brien S, Walsh T, Beverland DE. Modifiable factors delaying early discharge following primary joint arthroplasty. Eur J Orthop Surg Traumatol 2013; 23: 665-9.

6. Ilfeld BM. Continuous peripheral nerve blocks: a review of the published evidence. Anesth Analg 2011; 113: 904-25.

7. Capdevila X, Biboulet P, Bouregba M, Barthelet Y, Rubenovitch J, d'Athis F. Comparison of the three-in-one and fascia iliaca compartment blocks in adults: clinical and radiographic analysis. Anesth Analg 1998; 86: 1039-44.

8. Dolan J, Williams A, Murney E, Smith M, Kenny GN. Ultrasound guided fascia iliaca block: a comparison with the loss of resistance technique. Reg Anesth Pain Med 2008; 33: 526-31.

9. Prasad GA, Tumber PS, Lupu CM. Ultrasound guided spinal anesthesia. Can J Anaesth 2008; 55: 716-7.

10. Cushner F, Agnelli G, FitzGerald G, Warwick D. Complications and functional outcomes after total hip arthroplasty and total knee arthroplasty: results from the Global Orthopaedic Registry (GLORY). Am J Orthop (Belle Mead NJ) 2010; 39(9 Suppl): $22-8$.

11. Charous MT, Madison SJ, Suresh PJ, Sandhu NS, Loland VJ, Mariano ER, et al. Continuous femoral nerve blocks: varying local anesthetic 
delivery method (bolus versus basal) to minimize quadriceps motor block while maintaining sensory block. Anesthesiology 2011; 115: 774-81.

12. Mariano ER, Walters TL, Kim TE, Kain ZN. Why the perioperative surgical home makes sense for veterans affairs health care. Anesth Analg 2015; 120: 1163-6.

13. Mudumbai SC, Kim TE, Howard SK, Workman JJ, Giori N, Woolson S, et al. Continuous adductor canal blocks are superior to continuous femoral nerve blocks in promoting early ambulation after TKA. Clin Orthop Relat Res 2014; 472: 1377-83.

14. Benson K, Hartz AJ. A comparison of observational studies and randomized, controlled trials. N Engl J Med 2000; 342: $1878-86$.

15. Sturm R, Hattori A. Morbid obesity rates continue to rise rapidly in the United States. Int J Obes (Lond) 2013; 37: 889-91.

16. Singh JA, Sloan JA. Health-related quality of life in veterans with prevalent total knee arthroplasty and total hip arthroplasty. Rheumatology (Oxford) 2008; 47: 1826-31.

17. Choi S, Trang A, McCartney CJ. Reporting functional outcome after knee arthroplasty and regional anesthesia: a methodological primer. Reg Anesth Pain Med 2013; 38: 340-9.

18. Vincent HK, Weng JP, Vincent KR. Effect of obesity on inpatient rehabilitation outcomes after total hip arthroplasty. Obesity (Silver Spring) 2007; 15: 522-30.

19. Vincent HK, Vincent KR. Obesity and inpatient rehabilitation outcomes following knee arthroplasty: a multicenter study. Obesity (Silver Spring) 2008; 16: 130-6.

20. Tian W, DeJong G, Munin MC, Smout R. Patterns of rehabilitation after hip arthroplasty and the association with outcomes: an episode of care view. Am J Phys Med Rehabil 2010; 89: 905-18.

21. Pearse EO, Caldwell BF, Lockwood RJ, Hollard J. Early mobilisation after conventional knee replacement may reduce the risk of postoperative venous thromboembolism. J Bone Joint Surg Br 2007; 89: 316-22.

22. Vincent HK, DeJong G, Mascarenas D, Vincent KR. The effect of body mass index and hip abductor brace use on inpatient rehabilitation outcomes after total hip arthroplasty. Am J Phys Med Rehabil 2009; 88: 201-9.

23. Mahomed NN, Liang MH, Cook EF, Daltroy LH, Fortin PR, Fossel AH, et al. The importance of patient expectations in predicting functional outcomes after total joint arthroplasty. J Rheumatol 2002; 29: 1273-9.

24. Munin MC, Rudy TE, Glynn NW, Crossett LS, Rubash HE. Early inpatient rehabilitation after elective hip and knee arthroplasty. JAMA 1998; 279: 847-52.

25. Shariat AN, Hadzic A, Xu D, Shastri U, Kwofie K, Gandhi K, et al. Fascia lliaca block for analgesia after hip arthroplasty: a randomized double-blind, placebo-controlled trial. Reg Anesth Pain Med 2013; 38: 201-5.

26. Ilfeld BM, Mariano ER, Madison SJ, Loland VJ, Sandhu NS, Suresh PJ, et al. Continuous femoral versus posterior lumbar plexus nerve blocks for analgesia after hip arthroplasty: a randomized, controlled study. Anesth Analg 2011; 113: 897-903.

27. Ilfeld BM, Ball ST, Gearen PF, Le LT, Mariano ER, Vandenborne K, et al. Ambulatory continuous posterior lumbar plexus nerve blocks after hip arthroplasty: a dual-center, randomized, triple-masked, placebo-controlled trial. Anesthesiology 2008; 109: 491-501.

28. Horlocker TT, Wedel DJ, Rowlingson JC, Enneking FK, Kopp SL, Benzon HT, et al. Regional anesthesia in the patient receiving antithrombotic or thrombolytic therapy: American Society of Regional Anesthesia and Pain Medicine Evidence-Based Guidelines (Third Edition). Reg Anesth Pain Med 2010; 35: 64-101.

29. Memtsoudis SG, Sun X, Chiu YL, Stundner O, Liu SS, Banerjee S, et al. Perioperative comparative effectiveness of anesthetic technique in orthopedic patients. Anesthesiology 2013; 118: 1046-58.

30. Lopez S, Gros T, Bernard N, Plasse C, Capdevila X. Fascia iliaca compartment block for femoral bone fractures in prehospital care. Reg Anesth Pain Med 2003; 28: 203-7. 